\title{
Article
}

\section{Association between Lipid Levels and Risk for Different Types of Aneurysms: A Mendelian Randomization Study}

\author{
Yanghui Chen ${ }^{1,2}$, Man Huang ${ }^{1,2}$, Yunling Xuan ${ }^{1,2}, \mathrm{Ke} \mathrm{Li}^{1,2}$, Xin Xu ${ }^{1,2}$, Linlin Wang ${ }^{1,2}$, Yang Sun ${ }^{1,2}$, Lei Xiao ${ }^{1,2}$, \\ Ping $\mathrm{Xu}^{3}$, Wei Kong ${ }^{4}$ and Dao Wen Wang ${ }^{1,2, *}$ \\ 1 Division of Cardiology, Department of Internal Medicine, Tongji Hospital, Tongji Medical College, Huazhong \\ University of Science and Technology, Wuhan 430000, China; chenyh_c@163.com (Y.C.); \\ hm1127697727@163.com (M.H.); xylzzu@163.com (Y.X.); nikosrhee@163.com (K.L.); \\ mambaout8@163.com (X.X.); 13283931569@163.com (L.W.); sunyang.7@163.com (Y.S.); \\ xiaoleihust@163.com (L.X.) \\ 2 Hubei Key Laboratory of Genetics and Molecular Mechanism of Cardiologic Disorders, Huazhong University \\ of Science and Technology, Wuhan 430000, China \\ 3 Beijing Proteome Research Center, State Key Laboratory of Proteomics, National Center for Protein \\ Sciences (Beijing), Beijing Institute of Lifeomics, Beijing 100000, China; xupingghy@gmail.com \\ 4 Department of Physiology and Pathophysiology, School of Basic Medical Sciences, Peking University, \\ Beijing 100000, China; kongw@bjmu.edu.cn \\ * Correspondence: dwwang@tjh.tjmu.edu.cn; Tel./Fax: +86-027-83663280
}

check for updates

Citation: Chen, Y.; Huang, M.; Xuan, Y.; Li, K.; Xu, X.; Wang, L.; Sun, Y.;

Xiao, L.; Xu, P.; Kong, W.; et al.

Association between Lipid Levels and Risk for Different Types of Aneurysms: A Mendelian Randomization Study. J. Pers. Med. 2021, 11, 1171. https://doi.org/ 10.3390/jpm11111171

Academic Editor: David Alan Rizzieri

Received: 5 August 2021

Accepted: 8 November 2021

Published: 10 November 2021

Publisher's Note: MDPI stays neutral with regard to jurisdictional claims in published maps and institutional affiliations.

Copyright: (c) 2021 by the authors. Licensee MDPI, Basel, Switzerland. This article is an open access article distributed under the terms and conditions of the Creative Commons Attribution (CC BY) license (https:// creativecommons.org/licenses/by/ $4.0 /)$.
Abstract: Background: Although the associations between serum lipid levels and aneurysms have been investigated in epidemiological studies, causality remains unknown. Thus, this study aimed to investigate the causal relationships of serum high-density lipoprotein cholesterol (HDL-C), lowdensity lipoprotein cholesterol (LDL-C), total cholesterol (TC), and triglyceride (TG) levels on five types of aneurysms, using genetic variants associated with four lipid traits as instrumental variables in a Mendelian randomization (MR) analysis. Methods: We performed two-sample Mendelian randomization (MR) analyses to evaluate the associations of HDL-C, LDL-C, TC, and TG levels with risks for five types of aneurysms and those of LDL-C- (HMGCR, NPC1L1, PCSK9, CETP, and $L D L R$ ) and TG-lowering targets (ANGPTL3 and $L P L)$ with aneurysms. Results: The sample sizes of the included studies ranged from nearly 80,000 to 410,000 . We found inverse associations between genetically predicted HDL-C levels and aortic $(\mathrm{OR}=0.74,95 \% \mathrm{CI}=0.65-0.85)$ and abdominal aortic aneurysms $(0.58,0.45-0.75)$. A 1-SD increase in LDL-C and TC levels was associated with increased risks for aortic $(1.41,1.26-1.58$ and $1.36,1.18-1.56$, respectively) and abdominal aortic aneurysms (1.82, $1.48-2.22$ and $1.55,1.25-1.93$, respectively). TG levels were significantly associated with aortic (1.36, $1.18-1.56)$ and lower extremity artery aneurysms $(2.76,1.48-5.14)$, but limited to cerebral aneurysm $(1.23,1.06-1.42)$. Secondary analyses revealed a relationship between genetically proxied LDL-Clowering targets and all types of aneurysms; however, the drug targets remained heterogeneous. We found a weak association between TG-lowering therapies and aortic (ANGPTL3, 0.51, 0.29-0.89) and abdominal aortic aneurysms ( $L P L, 0.64,0.44-0.94)$. Conclusion: According to genetic evidence, lipid dysfunction is a causal risk factor for aneurysms. Lipid-lowering drugs may be a potential effective strategy in preventing and managing aneurysms.

Keywords: aneurysm; lipid traits; lipid-lowering drugs; mendelian randomization analyses

\section{Introduction}

Aneurysms, particularly cerebral and aortic aneurysms, are relatively rare but lifethreatening conditions associated with high mortality and morbidity rates. Although the relationship between serum lipid levels and risks for cardiocerebrovascular diseases, such as coronary heart disease, myocardial infarction, and stroke [1-4], has been well established, the relationship between serum lipid levels and aneurysms remains unclear. 
Different types of aneurysms share common risk factors and pathophysiological processes such as vascular remodeling, extracellular matrix degradation, and cell apoptosis. However, dysfunction of lipid metabolism across aneurysms is heterogeneous. In abdominal aortic aneurysm, several genetic studies revealed strong associations of serum high-density lipoprotein cholesterol (HDL-C) and low-density lipoprotein cholesterol (LDL-C) levels with risks for abdominal aortic aneurysms [5,6], and this trend was coincident with the observational studies [7]. However, in cerebral aneurysms, the role of lipid traits, particularly HDL-C, remains controversial. Elevated HDL-C levels contribute to a high risk of aneurysmal subarachnoid hemorrhage and ruptured cerebral aneurysms [8,9]; however, a large cohort of 6411 intracranial aneurysms suggested that higher HDL-C levels are inversely associated with rupture status [10]. Additionally, the current literature lacks studies regarding the role of lipid biomarkers in iliac artery aneurysm, and lower extremity artery aneurysm. In our study, not only abdominal aortic aneurysm but also other four different types of aneurysms were evaluated in terms of the association between lipid-associated single-nucleotide polymorphisms (SNPs) and risks. Additionally, we investigated the association between SNPs in lipid drug targets and risk of five types of aneurysms, which hopes to provide a potential effective treatment strategy for preventing and managing aneurysm.

Mendelian randomization (MR) is a popular approach in genetic epidemiology that circumvents the limitations of observational cohort studies [11]. In MR, genetic variants are usually used as instrumental variables to investigate causal associations, and MR has been widely used to explore the potential effect of exposure on disease [12-14]. Additionally, such MR analyses have been performed to determine whether licensed drugs or drug targets are causative $[15,16]$.

Therefore, in this study, we mainly investigated the causal relationship of serum HDL-C, LDL-C, total cholesterol (TC), and triglyceride (TG) levels on five types of aneurysms using genetic variants associated with four lipid traits as instrumental variables in an MR analysis. Furthermore, we assessed the association of LDL-C-lowering targets, including 3-hydroxy-3-methylglutaryl coenzyme A reductase (HMGCR), Niemann-pick C1-like 1 (NPC1L1), proprotein convertase subtilisin/kexin type 9 (PCSK9), cholesteryl ester transfer protein $(C E T P)$, and low-density lipoprotein receptor $(L D L R)$ and TG-lowering targets of angiopoietin-like 3 (ANGPTL3) and lipoprotein lipase ( $L P L)$ with aneurysms.

\section{Methods}

\subsection{Ethics Statement}

Our study was a secondary analysis of the publicly available data. Informed consent was sought for all participants per the original GWAS protocols, and all ethical approvals for the GWAS were obtained by the original GWAS authors.

\subsection{Study Design}

A two-sample MR analysis was conducted to examine the causal relationships of HDL-C, LDL-C, TG, and TC on the risks for various aneurysms, including cerebral aneurysm, aortic aneurysm, abdominal aortic aneurysm, iliac artery aneurysm, and lower extremity artery aneurysm. Electronic health records (ICD-9 or ICD-10 diagnosis and hospital procedure codes) from hospital episode statistics and summary statistics from publicly available genome-wide association studies (GWASs) were used. Secondary analyses were performed to determine the association of genetically proxied inhibition of lipid drug targets with aneurysm risks. The sample sizes of the included studies ranged from approximately 80,000 to 410,000, and all participants were of European ancestry. Participant characteristics and study details are shown in Table S1.

\subsection{Instrument Identification}

Summary data of lipid traits were obtained from a GWAS meta-analysis in the Global Lipids Genetics Consortium, which investigated HDL-C, LDL-C, TG, and TC levels of approximately 190,000 individuals [17]. For each lipid factor, the following inclusion criteria 
were defined as genetic instrument: (1) significant SNPs with exposure at $p<5 \times 10^{-8}$, (2) low-linkage disequilibrium (LD) (defined as $r^{2}<0.001$ ), (3) $F$-statistic $>10$, (4) significant SNPs with the outcome at $p>5 \times 10^{-8}$, and (5) validated by sensitivity analyses based on the MR pleiotropy residual sum and outlier (MR-PRESSO).

In the analyses of drug targets, all polymorphisms within a $\pm 100 \mathrm{~kb}$ window on target genes including HMGCR, NPC1L1, PCSK9, CETP, and LDLR, which are associated with LDL-C, and target genes including ANGPTL3 and LPL, which are associated with TG $\left(p<5 \times 10^{-8}\right)$, were identified. We conducted a clumping procedure to select independent SNPs at an LD $r^{2}$ threshold of 0.2 and a physical distance threshold of $250 \mathrm{~kb}$ [15].

\subsection{Summary-Level Genetic Data on Aneurysms}

The UK Biobank is a very large and detailed prospective study with over 500,000 participants aged 40-69 years recruited in 2006-2010. All participants provided informed consent to participate in the study and were required to complete a series of baseline measurements. GWAS summary statistics for cerebral aneurysm $(\mathrm{N}=408,164)$, aortic aneurysm $(\mathrm{N}=410,793)$, abdominal aortic aneurysm $(\mathrm{N}=409,983)$, aneurysm of iliac artery $(\mathrm{N}=408,718)$, and aneurysm of artery of lower extremity $(\mathrm{N}=408,811)$ were available in the pan-UKB (https: / / pan.ukbb.broadinstitute.org.2020, accessed on 19 March 2021) [18]. We also used data on aortic aneurysm $(\mathrm{N}=218,972)$ from the FinnGen R5 release. Additionally, Bakker's group performed a cross-ancestry, genome-wide association study in 10,754 cases and 306,882 controls of European and East Asian ancestry to discover new risk loci and the genetic architecture of cerebral aneurysms [19]. In our study, we downloaded the dataset from the stage 1 GWAS summary statistics comprising participants $(N=79,429)$ with European ancestry as an independent replication, as well.

\subsection{Two-Sample MR Analyses}

All MR analyses were conducted using R version 3.6.1 packages "TwoSampleMR" [20] and "MR-PRESSO" [21]. Effect estimates for single SNP were calculated using the Wald ratio method, i.e., by dividing the SNPs outcome association by the SNPs exposure association. The random-effects inverse variance weighted method, which provides a concise estimate and considers potential heterogeneity among estimates from individual variants, was performed to examine the association between multiple SNPs and outcomes [22]. For sensitivity analysis, we applied the following approaches: (1) MR-Egger regression method [23], which provided valid MR estimates in the presence of horizontal pleiotropy when the pleiotropic effects of the genetic variants were independent from the genetic associations with the exposure; (2) weighted median method [24], which provided valid MR estimates under the presence of horizontal pleiotropy when up to half of the included instruments were invalid; (3) weighted mode models [25], which were consistent when the largest number of similar single-variant MR estimates were derived from valid instruments. Additionally, we used $p<0.05 / 4$ exposures $/ 7$ outcomes $=0.0018$ to determine statistical significance.

As for multivariable MR analysis, a total of 211 SNPs were used in the analysis, which included SNPs which were genome-wide significant in one of HDL-C, LDL-C, and TG. The multivariable IVW method was utilized to assess the blood lipids' associations on aneurysms with the adjustment of other lipids.

To examine potential directional pleiotropy, we performed MR-Egger intercept analysis to test whether there was evidence of the intercept parameter being different from zero [23]. We also performed the global test, outlier test, and distortion test using MRPRESSO to detect outliers, which could bias the overall MR estimate [21]. For the analysis of cerebral and aortic aneurysm, statistical heterogeneity was assessed using Cochran Q chi-squared test (significant heterogeneity at $p<0.1$ and $I^{2}>50 \%$ ). To compare trials exhibiting heterogeneity, pooled data were meta-analyzed using a random-effects model; otherwise, a fixed-effects model was used. 


\section{Results}

\subsection{Genetic Instruments for Lipid Traits}

We obtained genetic instruments for four exposures (numbers of instruments: HDL-C $=88$, $\mathrm{LDL}-\mathrm{C}=79, \mathrm{TC}=86, \mathrm{TG}=55$ ) for MR analyses after excluding variants that were in $\mathrm{LD}$ $\left(r^{2}>0.01\right)$ and in proximity $(10 \mathrm{Mb})$ to other candidate instruments with stronger $p$-values (Table S2). The variance in each exposure explained by instrumental variables ranged from $5.6 \%$ to $9.6 \%$. All SNPs had $F$ values $>10$, suggesting that they were unlikely to introduce marked weak instrument bias in the MR analyses.

\subsection{Causal Estimates for Lipid Traits on Aneurysm Risks}

The results of the MR analyses investigating the associations between genetically predicted LDL-C, HDL-C, TC, and TG and aneurysm risk are presented in Table S3 and summarized in Figure 1.

\section{Cerebral aneurysm}

HDL-C
Bakker group
UK Biobank
Combind
LDL-C
Bakker group
UK Biobank
Combind
TC
Bakker group
UK Biobank
Combind
TG
Bakker group
UK Biobank
Combind

\begin{tabular}{|c|c|c|c|}
\hline SNP(N)/Methods & OR $(95 \% \mathrm{Cl})$ & & HDL-C \\
\hline 71 & $0.89(0.76-1.04)$ & 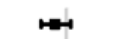 & FinnGen \\
\hline 88 & $0.75(0.50-1.12)$ & $\mapsto$ & UK Biobank \\
\hline Fixed & $0.87(0.75-1.00)$ & mer & $\begin{array}{l}\text { Combind } \\
\text { LDL-C }\end{array}$ \\
\hline 60 & $0.94(0.82-1.07)$ & Her & FinnGen \\
\hline 79 & $0.81(0.60-1.10)$ & $\mapsto$ & UK Biobank \\
\hline Fixed & $0.92(0.81-1.04)$ & 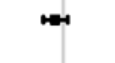 & $\begin{array}{l}\text { Combind } \\
\text { TC }\end{array}$ \\
\hline 64 & $0.95(0.85-1.06)$ & $\operatorname{men}$ & FinnGen \\
\hline 86 & $0.82(0.61-1.09)$ & $\mapsto$ & UK Biobank \\
\hline Fixed & $0.93(0.84-1.04)$ & $\operatorname{mos}$ & $\begin{array}{l}\text { Combind } \\
\text { TG }\end{array}$ \\
\hline 47 & $1.18(1.00-1.40)$ & $\mapsto$ & FinnGen \\
\hline 55 & $1.54(1.04-2.29)$ & $\longmapsto$ & UK Biobank \\
\hline Fixed & $1.23(1.06-1.42)$ & $\mapsto$ & Combind \\
\hline
\end{tabular}

Iliac artery aneurysm

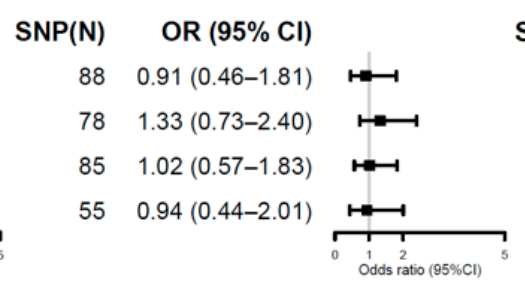

Aortic aneurysm

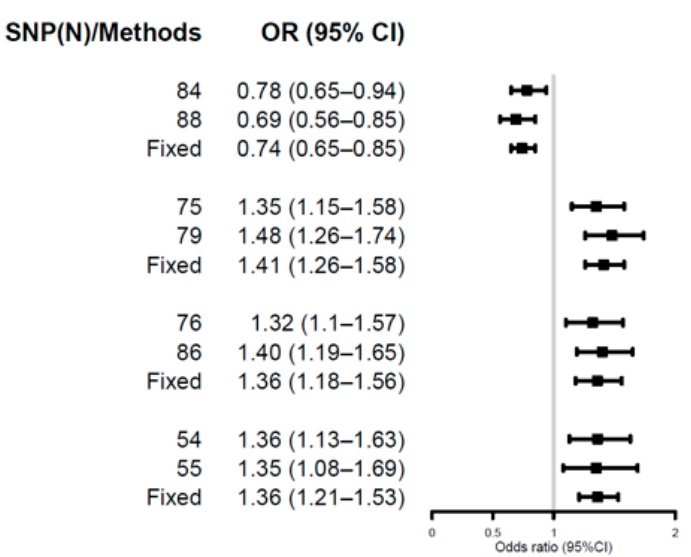

\section{Lower extremity artery aneurysm}

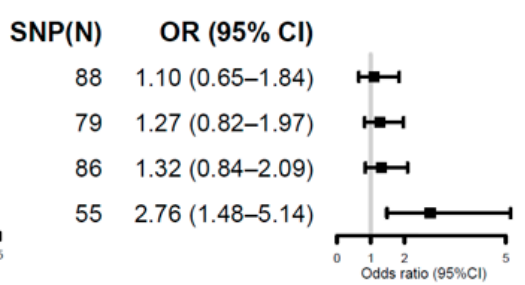

Figure 1. Forest plots showing inverse variance weighted method estimates (odds ratio with 95\% CI per 1 SD increase in lipid fraction) of HDL-C, LDL-C, TC, and TG single nucleotide polymorphisms on cerebral aneurysm, aortic aneurysm, abdominal aortic aneurysm, iliac artery aneurysm, and lower extremity artery aneurysm, respectively.

No associations were found between HDL-C ( $\mathrm{OR}=0.87,95 \% \mathrm{CI}=0.75-1, p=0.06)$, LDL-C (OR $=0.92,95 \% \mathrm{CI}=0.81-1.04, p=0.17)$, and TC (OR $=0.93,95 \% \mathrm{CI}=0.84-1.04$, $p=0.2)$ levels and cerebral aneurysms. Genetically higher TG levels showed suggestive evidence $(0.05<p<0.0018)$ for association with cerebral aneurysm $(\mathrm{OR}=1.23$, $95 \% \mathrm{CI}=1.06-1.42, p=0.006$ ).

All four lipid exposures were associated with aortic aneurysms after correction for multiple testing. We found significant inverse associations between genetically determined HDL-C levels and aortic aneurysms $(\mathrm{OR}=0.74,95 \% \mathrm{CI}=0.65-0.85)$. For $1-\mathrm{SD}$ increase in genetically predicted LDL-C, TC, and TG levels, the combined OR was 1.41 (95\% CI $=1.26-1.58), 1.36(95 \% \mathrm{CI}=1.18-1.56)$, and $1.36(95 \% \mathrm{CI}=1.21-1.53)$, respectively, for aortic aneurysms.

Genetically determined HDL-C, LDL-C, and TC levels were statistically associated with abdominal aortic aneurysms. A 1-SD increase in TG levels was nominally as- 
sociated with a $49 \%$ increase in overall abdominal aortic aneurysm risks (OR $=1.49$, $\left.95 \% \mathrm{CI}=1.13-1.97, p=4.8 \times 10^{-3}\right)$; however, this finding was not statistically significant after multiple testing correction.

No significant associations were noted between the four lipid traits and iliac artery aneurysms and lower extremity artery aneurysms, except TG levels. Genetically higher TG levels were significantly associated with an increased risk for lower extremity artery aneurysms $\left(\mathrm{OR}=2.76,95 \% \mathrm{CI}=1.48-5.14, p=1.3 \times 10^{-3}\right)$ but not for iliac artery aneurysms $(\mathrm{OR}=0.94,95 \% \mathrm{CI}=0.44-2.01, p=0.87)$.

In the multivariable MR analysis, the results showed HDL-C and LDL-C level remained associated with aortic aneurysms (HDL-C, OR $=0.82,95 \% \mathrm{CI}=0.73-0.93, p=2 \times 10^{-3}$; LDL-C, OR $=1.14,95 \% \mathrm{CI}=1.03-1.26, p=1 \times 10^{-2}$ ) and abdominal aortic aneurysms $\left(\mathrm{HDL}-\mathrm{C}, \mathrm{OR}=0.67,95 \% \mathrm{CI}=0.43-0.90, p=7.13 \times 10^{-4}\right.$; LDL-C, OR $=1.31,95 \% \mathrm{CI}=1.10-1.52$, $p=1.23 \times 10^{-2}$ ). Additionally, there was also evidence for a direct causal relationship of TG level on risk of cerebral aneurysm $\left(\mathrm{OR}=1.22,95 \% \mathrm{CI}=1.02-1.46, p=3 \times 10^{-2}\right)$ and lower extremity artery aneurysms $\left(\mathrm{OR}=2.16,95 \% \mathrm{CI}=1.50-2.81, p=2.08 \times 10^{-2}\right)$ (Table S4 and Figure S1).

\subsection{Genetic Proxies for Lipid Drug Targets and Aneurysm Risk}

The role of lipid levels in aneurysms has been well explored. We thus comprehensively assessed the causal relationships of genetic proxies for anti-lipid drugs on aneurysm risk. Details of the SNP effects are shown in Table S5, and the results are shown in Figures 2-4.

Cerebral aneurysm
UK Biobank
Bakker group
Combinded
Aortic aneurysm
FinnGen
UK Biobank
Combinded
Abdominal aortic aneurysm
UK Biobank
Iliac artery aneurysm
UK Biobank
Lower extremity artery aneurysm
UK Biobank

HMGCR

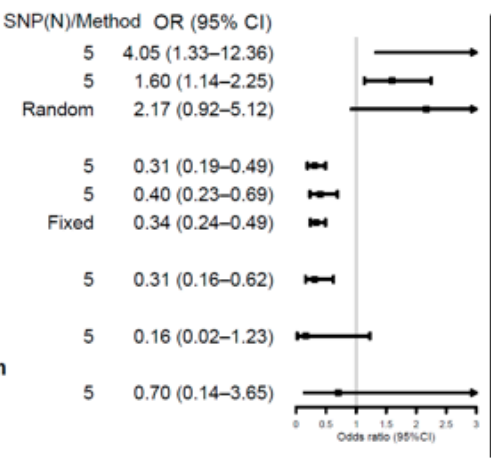

CETP

Cerebral aneurysm UK Biobank

Bakker group

Combinded

Aortic aneurysm

FinnGen

UK Biobank

Combinded

Abdominal aortic aneurysm

UK Biobank

Iliac artery aneurysm

UK Biobank

Lower extremity artery aneurysm

UK Biobank
SNP(N)/Method OR ( $95 \% \mathrm{Cl})$

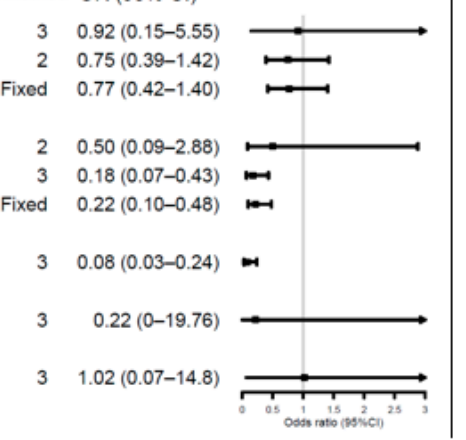

NPC1L1

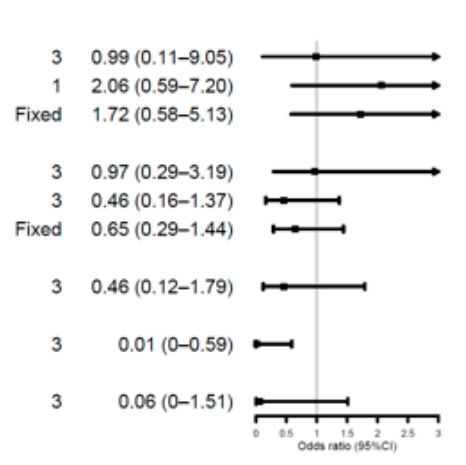

LDLR

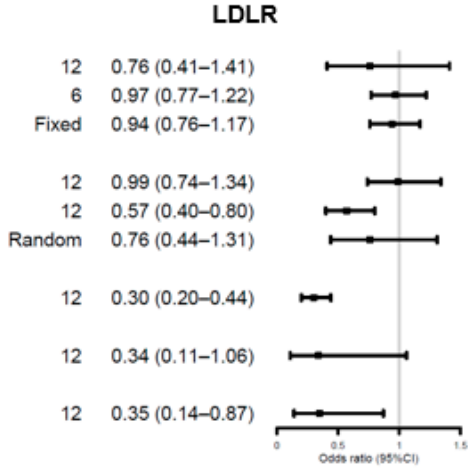

Figure 2. Forest plots showing inverse variance weighted method estimates (odds ratio with 95\% CI per $1 \mathrm{SD}$ increase in lipid fraction) of LDL-C-lowering HMGCR, NPC1L1, PCSK9, CETP, and LDLR single nucleotide polymorphisms on cerebral aneurysm, aortic aneurysm, abdominal aortic aneurysm, iliac artery aneurysm, and lower extremity artery aneurysm, respectively. 
ANGPTL3

Cerebral aneurysm
UK Biobank
Bakker group
Combinded
Aortic aneurysm
FinnGen
UK Biobank
Combinded
Abdominal aortic aneurysm
UK Biobank
Iliac artery aneurysm
UK Biobank
Lower extremity artery aneurysm
UK Biobank

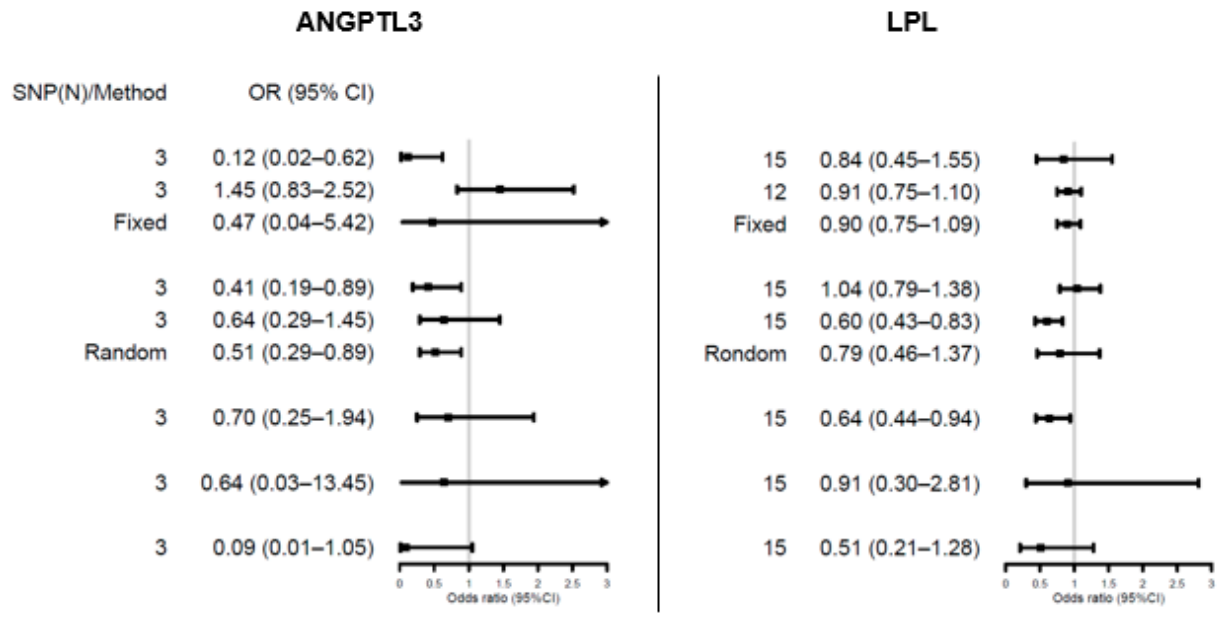

Figure 3. Forest plots showing inverse variance weighted method estimates (odds ratio with 95\% CI per 1 SD increase in lipid fraction) of TG-lowering ANGPTL3 and LPL single nucleotide polymorphisms on cerebral aneurysm, aortic aneurysm, abdominal aortic aneurysm, iliac artery aneurysm, and lower extremity artery aneurysm, respectively.

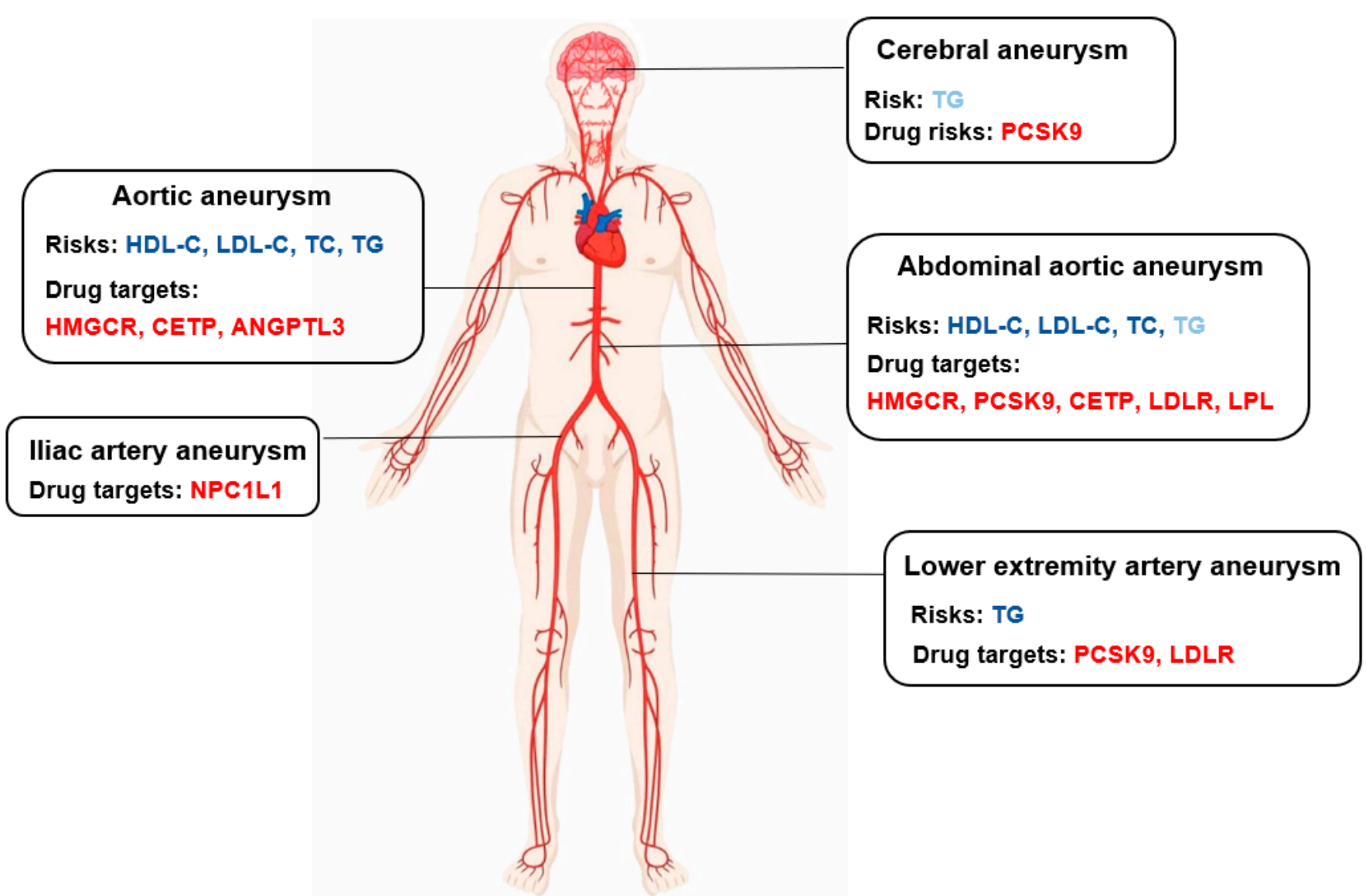

Figure 4. Lipid traits risk and lipid-lowering target in different aneurysms. Blue indicates the potential risk factors in aneurysms, and red indicates the potential protective factors in aneurysms. Dark blue means $p<0.0018$ for association on lipid levels by inverse variance weighted method and light blue means $p<0.05$. Red means $p<0.05$ for association on lipid-lowering target.

The MR analysis revealed an association between genetically proxied LDL-C-lowering inhibition of HMGCR with lower risks of aortic aneurysms $(\mathrm{OR}=0.34,95 \% \mathrm{CI}=0.24-0.49$, $\left.p=1 \times 10^{-5}\right)$ and abdominal aortic aneurysms $\left(\mathrm{OR}=0.31,95 \% \mathrm{CI}=0.16-0.62, p=8.51 \times 10^{-4}\right)$. A consistently strong risk association on cerebral aneurysms in the UK Biobank cohort 
$(\mathrm{OR}=4.05,95 \% \mathrm{CI}=1.33-12.36, p=0.014)$ and Bakker et al. $(\mathrm{OR}=1.6,95 \% \mathrm{CI}=1.14-2.25$, $\left.p=6.56 \times 10^{-3}\right)$ was noted, but with moderate heterogeneity between studies $\left(I^{2}=59 \%\right.$, random-effects model, $\mathrm{OR}=2.17,95 \% \mathrm{CI}=0.92-5.12, p=0.08)$.

Genetically proxied inhibition of NPC1L1 was not significantly associated with all types of aneurysms except for a weak association with lower iliac artery aneurysms $\left(\mathrm{OR}=0.01,95 \% \mathrm{CI}=0-0.59, p=2.69 \times 10^{-2}\right)$. Protective effects for PCSK9 were noted with respect to abdominal aortic aneurysms $\left(\mathrm{OR}=0.54,95 \% \mathrm{CI}=0.36-0.81, p=2.74 \times 10^{-3}\right)$ and lower extremity artery aneurysms $\left(\mathrm{OR}=0.12,95 \% \mathrm{CI}=0.04-0.4, p=5.52 \times 10^{-4}\right)$; however, the meta-analysis revealed a higher risk of cerebral aneurysm with respect to genetically proxied PCSK9 inhibition $(\mathrm{OR}=1.6,95 \% \mathrm{CI}=1.07-2.41, p=0.02)$. We found that genetically proxied PCSK9 could decrease the risk of aortic aneurysms in the UK Biobank cohort $\left(\mathrm{OR}=0.63,95 \% \mathrm{CI}=0.46-0.85, p=3.2 \times 10^{-3}\right)$; however, this was not replicated in the FinnGen cohort $(\mathrm{OR}=0.87,95 \% \mathrm{CI}=0.69-1.10, p=0.24)$.

CETP variants, resulting in genetically proxied reduction in LDL-C levels, reduced the risks of aortic aneurysms $\left(\mathrm{OR}=0.22,95 \% \mathrm{CI}=0.10-0.48, p=2 \times 10^{-4}\right)$ and abdominal aortic aneurysms $\left(\mathrm{OR}=0.08,95 \% \mathrm{CI}=0.03-0.24, p=7 \times 10^{-6}\right) . L D L R$ was associated with a reduction in risk for abdominal aortic aneurysms $(\mathrm{OR}=0.30,95 \% \mathrm{CI}=0.20-0.44$, $\left.p=4.54 \times 10^{-10}\right)$ and lower extremity artery aneurysms $(\mathrm{OR}=0.35,95 \% \mathrm{CI}=0.14-0.87$, $p=0.024)$.

Regarding the genetically proxied TG-lowering inhibition, ANGPTL3 variants were associated with lower risk of aortic aneurysms ( $\mathrm{OR}=0.51,95 \% \mathrm{CI}=0.29-0.89, p=0.02)$ and cerebral aneurysms in the UK Biobank cohort $(\mathrm{OR}=0.12,95 \% \mathrm{CI}=0.02-0.62, p=0.012)$. A similar finding was noted for $L P L$ variants on aortic aneurysms in the UK Biobank cohort $\left(\mathrm{OR}=0.60,95 \% \mathrm{CI}=0.43-0.83, p=1.95 \times 10^{-3}\right)$, but this was not replicated in the FinnGen cohort $(\mathrm{OR}=1.04,95 \% \mathrm{CI}=0.79-1.38, p=0.78) . L P L$ variants were also associated with risks for low abdominal aortic aneurysms $(\mathrm{OR}=0.64,95 \% \mathrm{CI}=0.44-0.94, p=0.022)$.

\section{Discussion}

In this study, we systematically evaluated the causality of the association between four lipid traits (HDL-C, LDL-C, TC, and TG) and aneurysm risks using two-sample MR analyses and found associations between genetically predicted HDL-C, LDL-C, and TC levels and aortic and abdominal aortic aneurysms. TG levels were significantly associated with aortic and lower extremity artery aneurysms, but limited to cerebral aneurysm.

\subsection{Controversial Role of Lipid TRAITS in Cerebral Aneurysms}

The role of cholesterol on the risk for cerebral aneurysm is unclear. In this study, we did not observe any significant associations of genetically predicted HDL-C, LDL-C, and TC levels with cerebral aneurysms. Similarly, the association between cholesterol-lowering drugs and cerebral aneurysms is controversial. Retrospective studies reported that statin use (HMGCR inhibitor) is inversely associated with cerebral aneurysm rupture $[10,26]$; however, no strong significant beneficial effect or even increased risk of side effects has been noted in clinical trials $[27,28]$.

Previous studies showed PCSK9 inhibitors did not reduce the risk of brain stroke, but newly large clinical studies, such as FOURIER trials, reported its beneficial effects on brain stroke and ischemic brain stroke [29], which might not corresponded to the effect size observed in this analysis. In our study, we found that the inhibition of genetically proxied LDL-C-lowering targets, PCSK9, might increase the risk of cerebral aneurysm. This might be because MR estimated the long-term modulation of drug target on the disease risk, and the most relevant limitation of clinical trials is that patients were followed for a relatively short period of time, which might limit definitive conclusions on the long-term effects of extreme cholesterol lowering induced by PCSK9 inhibitors. 


\subsection{Lipid Dysfunction in Aortic Aneurysms: From Etiology to Drug Therapy}

Aortic aneurysms are potentially lethal conditions that cause more than 10,000 deaths per year in the United Kingdom and United States [30,31]. Unfortunately, there is a lack of effective medications for preventing disease progression. Genetic studies have reported some abdominal aortic aneurysm risk loci associated with lipoprotein metabolism [32]. In this study, we provided robust evidence that lipid dysfunction, mainly that of higher HDL-C levels, lower LDL-C, TC, and TG levels, may play a causal role in the etiology of aortic/abdominal aortic aneurysms, and HDL-C and LDL-C level remained associated with aortic aneurysms and abdominal aortic aneurysms after multivariable MR analysis, which was consistent with results of previous observational studies [33,34].

From our analysis, we suggest that lower LDL-C treatment (such as HMGCR inhibitor) might be beneficial to patients. It had been demonstrated that statin use was strongly recommended for patients undergoing vascular surgery, including abdominal aortic aneurysms [35], because statin use could significantly decrease the perioperative morbidity and mortality rates (perioperative strokes, myocardial infarctions, and cardiovascular deaths) [36]. A recent meta-analysis explored the effects of statin use on the outcomes of abdominal aortic aneurysms, and found statin use was associated with significant reductions in long-term and short-term mortality rates [37].

PSCK9 inhibitor is another potential lower LDL-C treatment which was associated with lower odds of abdominal aortic aneurysms from our study. Although there is a lack of clinical trials exploring the effect in aortic aneurysms patients, we believed PCSK9 inhibitor was beneficial to abdominal aortic aneurysm patients. Firstly, PCSK9 plays a critical role in the pathogenesis of vascular diseases. It induces smooth muscle cell dedifferentiation and LDL-C level upregulation via LDLR degradation and upregulates extracellular matrix degradation and adipocytokine expression [38-40]. Secondly, the results of recent FOURIER trial showed that adding evolocumab (PCSK9 inhibitor) to statin could reduce the risk of acute arterial events across all vascular territories [29].

Apart from HMGCR and PCSK9 inhibitions, ANGPTL3 might be another potential target for the treatment of aortic aneurysms in our study. To date, targeting ANGPTL3 were emerging in clinical recently, such as evinacumab (a human monoclonal antibody of ANGPTL3) and Antisense oligonucleotides (ASO)-based inhibition of ANGPTL3. These studies showed that ANGPTL3 inhibition resulted in a favorable lipid profile (lower TG and LDL-C levels) and provided potential protection in atherosclerotic cardiovascular diseases $[41,42]$. There are data to support its beneficial effects on vascular remodeling that could have relevance in aortic aneurysms management [43].

\subsection{Limited Evidence on Other Aneurysms}

Iliac artery aneurysm is a rare vascular disease, but its rupture is associated with high mortality rates. Its main cause is related to progressive atherosclerosis [44]; however, the role of lipid values in the pathogenesis and pathophysiology of iliac artery aneurysms remain unclear. Although we did not observe lipid dysfunction, we speculate that the NPC1L1 inhibitor likely mitigates the risk of iliac artery aneurysms via an unknown mechanism that might be related to an NPC1L1-specific pathway, which differs from the LDLR pathway.

\section{Limitations}

This study had several limitations. First, although MR had features that minimized the risk for confounding and reverse causation, it was hard to eliminate all the potential influence of directional pleiotropy, causing biased causal effect estimates. In our study, we attempted to control for pleiotropy by using different sensitivity analyses (MR-Egger, weighted median, and weighted mode analyses), but pleiotropic lipid-based pathway still might be represented as the SNPs were connected within the metabolite network and therefore not independent of each other. Second, the Bonferroni correction for multiple tests performed in the sensitivity analyses suggests that some findings may be false positives. 
Third, our analyses were based on data obtained from the European population, thus limiting the generalizability of our results to other ethnicities. Finally, although we did not observe any association between lipid traits and iliac artery aneurysms, the negative findings might be attributed to a lack of sufficient statistical power to detect an effect.

\section{Conclusions}

In conclusion, we found genetic evidence to support higher LDL-C, TC, and lower HDL-C as a causal risk factor for aortic aneurysm and abdominal aortic aneurysm. Higher TG levels were significantly associated with aortic and lower extremity artery aneurysms, but modestly associated with cerebral aneurysm. In addition to this, interventions that lower LDL-C are likely to be beneficial for all types of aneurysms expect cerebral aneurysm. TG-lowering therapies were associated with lower odds of aortic and abdominal aortic aneurysms.

Supplementary Materials: The following are available online at https://www.mdpi.com/article/10.339 0/jpm11111171/s1. Figure S1: Forest plots showing multivariable MR estimates (odds ratio with 95\% CI per 1 SD increase in lipid fraction) of HDL-C, LDL-C, and TG single nucleotide polymorphisms on cerebral aneurysm, aortic aneurysm, abdominal aortic aneurysm, iliac artery aneurysm and lower extremity artery aneurysm, respectively. Table S1: summary of Genome-Wide Association Studies involved in our study. Table S2: Detailed summary of genetic variants in HDL-C, LDL-C, TC and TG. Table S3: All Mendelian randomization analysis results of lipid traits. Table S4: Multivariable mendelian randomization analysis results of lipid traits with adjustment for HDL-C, LDL-C and TG. Table S5: Detailed summary of LDL-C lowering genetic variants in HMGCR, NPC1L1, CETP, PCSK9, LDLR and TG lowering genetic variants in ANGPTL3, LPL.

Author Contributions: Data curation, Y.C., M.H. and K.L.; Formal analysis, Y.C.; Funding acquisition, P.X., W.K. and D.W.W.; Investigation, Y.X., X.X., L.W., L.X. and D.W.W.; Methodology, Y.C.; Project administration, D.W.W.; Software, M.H., Y.X., K.L. and L.W.; Validation, Y.S.; Visualization, X.X. and L.X.; Writing—original draft, Y.C.; Writing—review \& editing, Y.S. All authors have read and agreed to the published version of the manuscript.

Funding: This work was supported by National Natural Science Foundation of China projects (NO. 91839302), National Key R\&D Program of China (NO. 2017YFC0909400) and Shanghai Municipal Science and Technology Major Project (Grant No. 2017SHZDZX01).

Institutional Review Board Statement: Not applicable.

Informed Consent Statement: Not applicable.

Data Availability Statement: The data are available in Supplementary Files and upon request.

Acknowledgments: We thank the Global Lipids Genetics Consortium, UK Biobank, the Pan-UKB team, the participants and investigators of FinnGen study, Mark K. Bakker team for making summary statistics publicly accessible for this analysis.

Conflicts of Interest: There are no conflict of interest to declare.

\section{References}

1. Ference, B.A.; Ginsberg, H.N.; Graham, I.; Ray, K.K.; Packard, C.J.; Bruckert, E.; Hegele, R.A.; Krauss, R.M.; Raal, F.J.; Schunkert, H.; et al. Low-density lipoproteins cause atherosclerotic cardiovascular disease. 1. Evidence from genetic, epidemiologic, and clinical studies. A consensus statement from the European Atherosclerosis Society Consensus Panel. Eur. Heart J. 2017, 38, 2459-2472. [CrossRef]

2. Di Angelantonio, E.; Gao, P.; Pennells, L.; Kaptoge, S.; Caslake, M.; Thompson, A.; Butterworth, A.S.; Sarwar, N.; Wormser, D.; Emerging Risk Factors Collaboration; et al. Lipid-related markers and cardiovascular disease prediction. JAMA 2012, 307, 2499-2506. [CrossRef]

3. Holmes, M.V.; Millwood, I.Y.; Kartsonaki, C.; Hill, M.R.; Bennett, D.A.; Boxall, R.; Guo, Y.; Xu, X.; Bian, Z.; Hu, R.; et al. Lipids, Lipoproteins, and Metabolites and Risk of Myocardial Infarction and Stroke. J. Am. Coll. Cardiol. 2018, 71, 620-632. [CrossRef] [PubMed]

4. Rist, P.M.; Buring, J.E.; Ridker, P.M.; Kase, C.S.; Kurth, T.; Rexrode, K.M. Lipid levels and the risk of hemorrhagic stroke among women. Neurology 2019, 92, e2286-e2294. [CrossRef] [PubMed] 
5. Harrison, S.C.; Holmes, M.V.; Burgess, S.; Asselbergs, F.W.; Jones, G.T.; Baas, A.F.; van ‘t Hof, F.N.; de Bakker, P.I.W.; Blankensteijn, J.D.; Powell, J.T.; et al. Genetic Association of Lipids and Lipid Drug Targets With Abdominal Aortic Aneurysm: A Meta-analysis. JAMA Cardiol. 2018, 3, 26-33. [CrossRef]

6. Allara, E.; Morani, G.; Carter, P.; Gkatzionis, A.; Zuber, V.; Foley, C.N.; Rees, J.M.B.; Mason, A.M.; Bell, S.; Gill, D.; et al. Genetic Determinants of Lipids and Cardiovascular Disease Outcomes: A Wide-Angled Mendelian Randomization Investigation. Circ. Genom. Precis. Med. 2019, 12, e002711. [CrossRef] [PubMed]

7. Golledge, J.; van Bockxmeer, F.; Jamrozik, K.; McCann, M.; Norman, P.E. Association between serum lipoproteins and abdominal aortic aneurysm. Am. J. Cardiol. 2010, 105, 1480-1484. [CrossRef] [PubMed]

8. Zhang, Y.; Tuomilehto, J.; Jousilahti, P.; Wang, Y.; Antikainen, R.; Hu, G. Total and high-density lipoprotein cholesterol and stroke risk. Stroke 2012, 43, 1768-1774. [CrossRef]

9. Huang, Q.; Shang-Guan, H.C.; Wu, S.Y.; Yao, P.S.; Sun, Y.; Zeng, Y.L.; Zheng, S.F.; Chen, G.R.; Lin, Y.X.; Kang, D.Z. High-Density Lipoprotein Is Associated with Progression of Intracranial Aneurysms. World Neurosurg. 2018, 120, e234-e240. [CrossRef]

10. Can, A.; Castro, V.M.; Dligach, D.; Finan, S.; Yu, S.; Gainer, V.; Shadick, N.A.; Savova, G.; Murphy, S.; Cai, T.; et al. Lipid-Lowering Agents and High HDL (High-Density Lipoprotein) Are Inversely Associated With Intracranial Aneurysm Rupture. Stroke 2018, 49, 1148-1154. [CrossRef]

11. Smith, G.D.; Ebrahim, S. Mendelian randomization: Can genetic epidemiology contribute to understanding environmental determinants of disease? Int. J. Epidemiol. 2003, 32, 1-22. [CrossRef]

12. Lawlor, D.A.; Harbord, R.M.; Sterne, J.A.; Timpson, N.; Smith, G.D. Mendelian randomization: Using genes as instruments for making causal inferences in epidemiology. Stat. Med. 2008, 27, 1133-1163. [CrossRef]

13. Leong, A.; Cole, J.B.; Brenner, L.N.; Meigs, J.B.; Florez, J.C.; Mercader, J.M. Cardiometabolic risk factors for COVID-19 susceptibility and severity: A Mendelian randomization analysis. PLoS Med. 2021, 18, e1003553. [CrossRef]

14. Ponsford, M.J.; Gkatzionis, A.; Walker, V.M.; Grant, A.J.; Wootton, R.E.; Moore, L.S.P.; Fatumo, S.; Mason, A.M.; Zuber, V.; Willer, C.; et al. Cardiometabolic Traits, Sepsis, and Severe COVID-19: A Mendelian Randomization Investigation. Circulation 2020, 142, 1791-1793. [CrossRef] [PubMed]

15. Yarmolinsky, J.; Bull, C.J.; Vincent, E.E.; Robinson, J.; Walther, A.; Smith, G.D.; Lewis, S.J.; Relton, C.L.; Martin, R.M. Association Between Genetically Proxied Inhibition of HMG-CoA Reductase and Epithelial Ovarian Cancer. JAMA 2020, 323, 646-655. [CrossRef] [PubMed]

16. Gaziano, L.; Giambartolomei, C.; Pereira, A.C.; Gaulton, A.; Posner, D.C.; Swanson, S.A.; Ho, Y.L.; Iyengar, S.K.; Kosik, N.M.; Vujkovic, M.; et al. Actionable druggable genome-wide Mendelian randomization identifies repurposing opportunities for COVID-19. Nat. Med. 2021, 27, 668-676. [CrossRef]

17. Willer, C.J.; Schmidt, E.M.; Sengupta, S.; Peloso, G.M.; Gustafsson, S.; Kanoni, S.; Ganna, A.; Chen, J.; Buchkovich, M.L.; Mora, S.; et al. Discovery and refinement of loci associated with lipid levels. Nat. Genet. 2013, 45, 1274-1283. [CrossRef] [PubMed]

18. Sudlow, C.; Gallacher, J.; Allen, N.; Beral, V.; Burton, P.; Danesh, J.; Downey, P.; Elliott, P.; Green, J.; Landray, M.; et al. UK biobank: An open access resource for identifying the causes of a wide range of complex diseases of middle and old age. PLoS Med. 2015, 12, e1001779. [CrossRef] [PubMed]

19. Bakker, M.K.; van der Spek, R.A.A.; van Rheenen, W.; Morel, S.; Bourcier, R.; Hostettler, I.C.; Alg, V.S.; van Eijk, K.R.; Koido, M.; Akiyama, M.; et al. Genome-wide association study of intracranial aneurysms identifies 17 risk loci and genetic overlap with clinical risk factors. Nat. Genet. 2020, 52, 1303-1313. [CrossRef]

20. Yavorska, O.O.; Burgess, S. MendelianRandomization: An R package for performing Mendelian randomization analyses using summarized data. Int. J. Epidemiol. 2017, 46, 1734-1739. [CrossRef]

21. Verbanck, M.; Chen, C.Y.; Neale, B.; Do, R. Detection of widespread horizontal pleiotropy in causal relationships inferred from Mendelian randomization between complex traits and diseases. Nat. Genet. 2018, 50, 693-698. [CrossRef] [PubMed]

22. Burgess, S.; Dudbridge, F.; Thompson, S.G. Combining information on multiple instrumental variables in Mendelian randomization: Comparison of allele score and summarized data methods. Stat. Med. 2016, 35, 1880-1906. [CrossRef] [PubMed]

23. Bowden, J.; Smith, G.D.; Burgess, S. Mendelian randomization with invalid instruments: Effect estimation and bias detection through Egger regression. Int. J. Epidemiol. 2015, 44, 512-525. [CrossRef] [PubMed]

24. Bowden, J.; Smith, G.D.; Haycock, P.C.; Burgess, S. Consistent Estimation in Mendelian Randomization with Some Invalid Instruments Using a Weighted Median Estimator. Genet. Epidemiol. 2016, 40, 304-314. [CrossRef]

25. Hartwig, F.P.; Smith, G.D.; Bowden, J. Robust inference in summary data Mendelian randomization via the zero modal pleiotropy assumption. Int. J. Epidemiol. 2017, 46, 1985-1998. [CrossRef]

26. Yoshimura, Y.; Murakami, Y.; Saitoh, M.; Yokoi, T.; Aoki, T.; Miura, K.; Ueshima, H.; Nozaki, K.; SSS Research Group. Statin use and risk of cerebral aneurysm rupture: A hospital-based case-control study in Japan. J. Stroke Cereb. Dis. 2014, 23, 343-348. [CrossRef]

27. Naraoka, M.; Matsuda, N.; Shimamura, N.; Asano, K.; Akasaka, K.; Takemura, A.; Hasegawa, S.; Ohkuma, H. Long-acting statin for aneurysmal subarachnoid hemorrhage: A randomized, double-blind, placebo-controlled trial. J. Cereb. Blood Flow Metab. 2018, 38, 1190-1198. [CrossRef]

28. Wong, G.K.; Chan, D.Y.; Siu, D.Y.; Zee, B.C.; Poon, W.S.; Chan, M.T.; Gin, T.; Leung, M.; Investigators, H.-S. High-dose simvastatin for aneurysmal subarachnoid hemorrhage: Multicenter randomized controlled double-blinded clinical trial. Stroke 2015, 46, 382-388. [CrossRef] 
29. Oyama, K.; Giugliano, R.P.; Tang, M.; Bonaca, M.P.; Saver, J.L.; Murphy, S.A.; Ruzza, A.; Keech, A.C.; Sever, P.S.; Sabatine, M.S.; et al. Effect of evolocumab on acute arterial events across all vascular territories: Results from the FOURIER trial. Eur. Heart $J$. 2021, ehab604. [CrossRef]

30. McPhee, J.T.; Hill, J.S.; Eslami, M.H. The impact of gender on presentation, therapy, and mortality of abdominal aortic aneurysm in the United States, 2001-2004. J. Vasc. Surg. 2007, 45, 891-899. [CrossRef]

31. Ashton, H.A.; Buxton, M.J.; Day, N.E.; Kim, L.G.; Marteau, T.M.; Scott, R.A.; Thompson, S.G.; Walker, N.M.; Multicentre Aneurysm Screening Study Group. The Multicentre Aneurysm Screening Study (MASS) into the effect of abdominal aortic aneurysm screening on mortality in men: A randomised controlled trial. Lancet 2002, 360, 1531-1539. [CrossRef]

32. Jones, G.T.; Tromp, G.; Kuivaniemi, H.; Gretarsdottir, S.; Baas, A.F.; Giusti, B.; Strauss, E.; Van't Hof, F.N.; Webb, T.R.; Erdman, R.; et al. Meta-Analysis of Genome-Wide Association Studies for Abdominal Aortic Aneurysm Identifies Four New Disease-Specific Risk Loci. Circ. Res. 2017, 120, 341-353. [CrossRef]

33. Tang, W.; Yao, L.; Roetker, N.S.; Alonso, A.; Lutsey, P.L.; Steenson, C.C.; Lederle, F.A.; Hunter, D.W.; Bengtson, L.G.; Guan, W.; et al. Lifetime Risk and Risk Factors for Abdominal Aortic Aneurysm in a 24-Year Prospective Study: The ARIC Study (Atherosclerosis Risk in Communities). Arter. Thromb. Vasc. Biol. 2016, 36, 2468-2477. [CrossRef] [PubMed]

34. Forsdahl, S.H.; Singh, K.; Solberg, S.; Jacobsen, B.K. Risk factors for abdominal aortic aneurysms: A 7-year prospective study: The Tromso Study, 1994-2001. Circulation 2009, 119, 2202-2208. [CrossRef]

35. Chaikof, E.L.; Dalman, R.L.; Eskandari, M.K.; Jackson, B.M.; Lee, W.A.; Mansour, M.A.; Mastracci, T.M.; Mell, M.; Murad, M.H.; Nguyen, L.L.; et al. The Society for Vascular Surgery practice guidelines on the care of patients with an abdominal aortic aneurysm. J. Vasc. Surg. 2018, 67, 2-77. [CrossRef] [PubMed]

36. Lindenauer, P.K.; Pekow, P.; Wang, K.; Gutierrez, B.; Benjamin, E.M. Lipid-lowering therapy and in-hospital mortality following major noncardiac surgery. JAMA 2004, 291, 2092-2099. [CrossRef]

37. Xiong, X.; Wu, Z.; Qin, X.; Huang, Q.; Wang, X.; Qin, J.; Lu, X. Statins reduce mortality after abdominal aortic aneurysm repair: A systematic review and meta-analysis. J. Vasc. Surg. 2021. [CrossRef] [PubMed]

38. Lu, H.; Howatt, D.A.; Balakrishnan, A.; Graham, M.J.; Mullick, A.E.; Daugherty, A. Hypercholesterolemia Induced by a PCSK9 Gain-of-Function Mutation Augments Angiotensin II-Induced Abdominal Aortic Aneurysms in C57BL/6 Mice-Brief Report. Arter. Thromb. Vasc. Biol. 2016, 36, 1753-1757. [CrossRef]

39. Ding, Z.; Liu, S.; Wang, X.; Deng, X.; Fan, Y.; Sun, C.; Wang, Y.; Mehta, J.L. Hemodynamic shear stress via ROS modulates PCSK9 expression in human vascular endothelial and smooth muscle cells and along the mouse aorta. Antioxid. Redox Signal. 2015, 22, 760-771. [CrossRef] [PubMed]

40. Guo, Y.; Yan, B.; Gui, Y.; Tang, Z.; Tai, S.; Zhou, S.; Zheng, X.L. Physiology and role of PCSK9 in vascular disease: Potential impact of localized PCSK9 in vascular wall. J. Cell. Physiol. 2021, 236, 2333-2351. [CrossRef]

41. Dewey, F.E.; Gusarova, V.; Dunbar, R.L.; O’Dushlaine, C.; Schurmann, C.; Gottesman, O.; McCarthy, S.; Van Hout, C.V.; Bruse, S.; Dansky, H.M.; et al. Genetic and Pharmacologic Inactivation of ANGPTL3 and Cardiovascular Disease. N. Engl. J. Med. 2017, 377, 211-221. [CrossRef] [PubMed]

42. Harada-Shiba, M.; Ali, S.; Gipe, D.A.; Gasparino, E.; Son, V.; Zhang, Y.; Pordy, R.; Catapano, A.L. A randomized study investigating the safety, tolerability, and pharmacokinetics of evinacumab, an ANGPTL3 inhibitor, in healthy Japanese and Caucasian subjects. Atherosclerosis 2020, 314, 33-40. [CrossRef] [PubMed]

43. Ye, Q.; Tian, G.P.; Cheng, H.P.; Zhang, X.; Ou, X.; Yu, X.H.; Tan, R.Q.; Yang, F.Y.; Gong, D.; Huang, C.; et al. MicroRNA-134 Promotes the Development of Atherosclerosis Via the ANGPTL4/LPL Pathway in Apolipoprotein E Knockout Mice. J. Atheroscler. Thromb. 2018, 25, 244-253. [CrossRef] [PubMed]

44. Dix, F.P.; Titi, M.; Al-Khaffaf, H. The isolated internal iliac artery aneurysm-A review. Eur. J. Vasc. Endovasc. Surg. 2005, 30, 119-129. [CrossRef] [PubMed] 